\title{
Gradhiva
}

GRADHIV

Revue d'anthropologie et d'histoire des arts

$22 \mid 2015$

Cosmos

\section{Astromorphing. Des planètes, des visages et des ondes de longue portée en astrologie}

Astromorphing. On planets, faces and long-range waves in astrology

\section{Emmanuel Grimaud}

\section{(2) OpenEdition}

\section{Journals}

\section{Édition électronique}

URL : http://journals.openedition.org/gradhiva/3033

DOI : 10.4000/gradhiva.3033

ISSN : 1760-849X

Éditeur

Musée du quai Branly Jacques Chirac

\section{Édition imprimée}

Date de publication : 1 octobre 2015

Pagination : 49-73

ISBN : 978-2-35744-092-0

ISSN : 0764-8928

\section{Référence électronique}

Emmanuel Grimaud, «Astromorphing. Des planètes, des visages et des ondes de longue portée en astrologie », Gradhiva [En ligne], 22 | 2015, mis en ligne le 01 octobre 2018, consulté le 21 avril 2019 URL : http://journals.openedition.org/gradhiva/3033; DOI : 10.4000/gradhiva.3033

(c) musée du quai Branly 
र 9 r

निसर्गान

सर्वजितनाम संवृसरे सुर्योद्य-ह-22 चरण. तुक्ष याषी

मद्रास $\varepsilon-\gamma$

की

काहे *ैं

च्याप्सरेच-।

नास नांही वाचां.

परका अात्तानिधास-

चतेमुल्ठ अनुपम आंखे

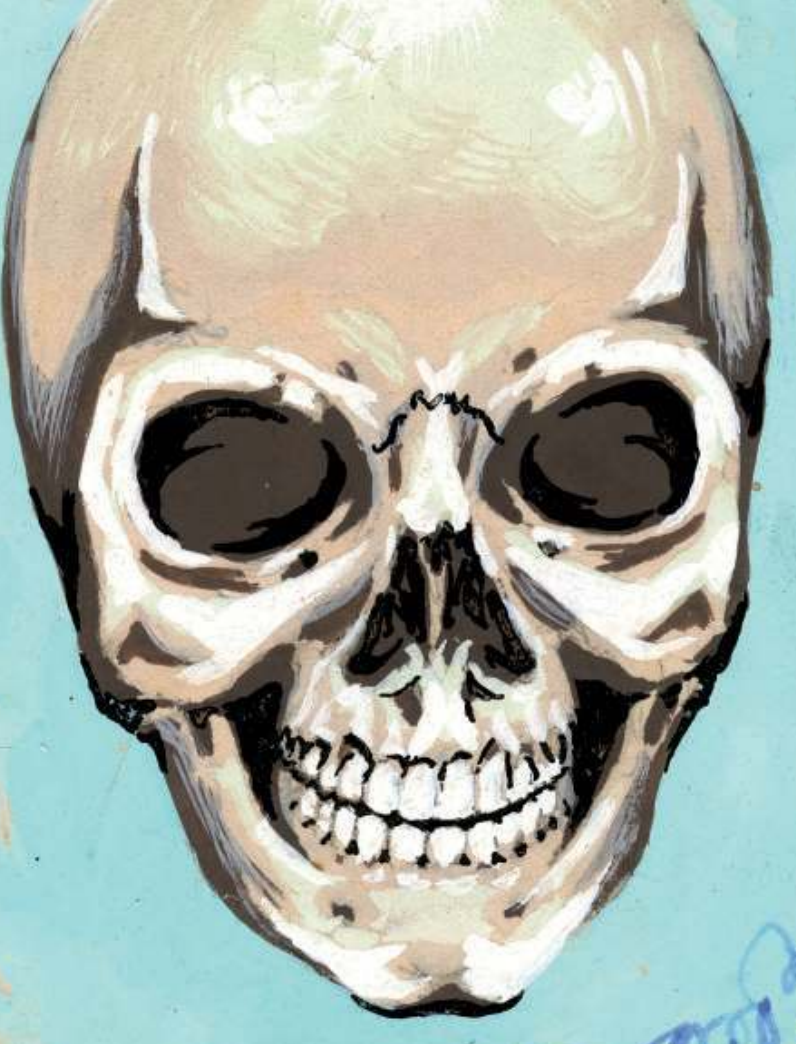

ति तच्वात आज्याने

व्यक्त करणारी असा

$2 \% 0.70$

थानाचा आहे मृणून

नाही- एका सरकारी

स येकून $? \xi व$ वर्षा

रिक्षिणघंकन व्यौत 


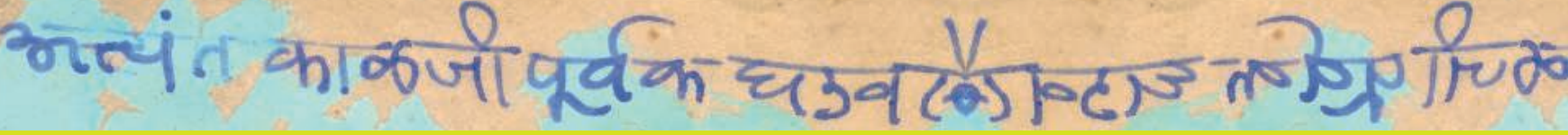

Astromorphing

Des planètes, des visages et des ondes de longue portée en astrologie

par Emmanuel Grimaud

L'astrologie possède sans doute en Inde son plus grand laboratoire, si l'on en croit l'éventail exceptionnel de situations dans lesquelles elle se trouve mobilisée. Elle jouit par ailleurs d'une reconnaissance institutionnelle, donnant lieu à des enseignements et des recherches très variées, au croisement de la science, de la médecine et de la divination. Contrairement aux idées reçues, elle possède une dimension expérimentale indéniable. Elle n'a jamais cessé de se réinventer au cours du temps, donnant lieu à de nouvelles formes de connexion aux astres, à un affinement des méthodes de calcul pour anticiper leurs fluctuations et à de nouveaux procédés pour magnétiser autrement tout ce qui nous entoure. C'est plus particulièrement à l'astromorphologie que cet article est consacré et aux techniques utilisées par un astrologue indien pour mesurer les emprises des astres à même le visage et leurs mécanismes d'influence à distance. La consultation astrologique est envisagée ici comme un lieu privilégié où faire des expériences en résonance dont on peut suivre en situation les enchaînements et les implications sur les personnes, modifiant leur rapport au corps et à l'environnement (astromorphose). 


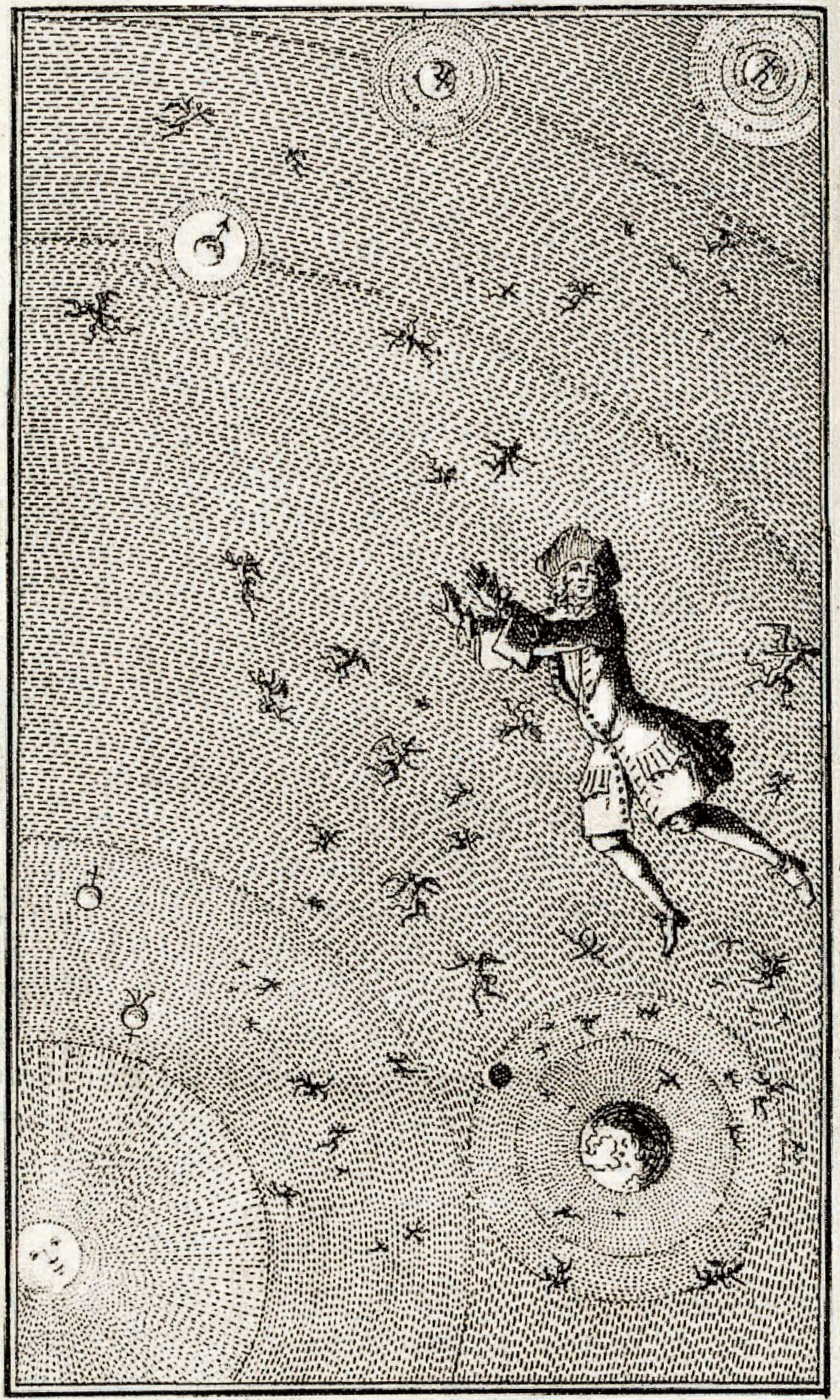

-VISION DU MONDE ANGELIQUE. 
«Le corps physique est sous l'emprise de la gravité. La gravité de notre mère la Terre rassemble tous les êtres qui se développent en son sein. Comme les personnalités humaines résident dans des corps physiques, les corps planétaires sont comme des corps physiques pour les neuf divinités qui ont la plus grande emprise. À l'exception de la Lune et du Soleil, la gravité physique des planètes affecte les créatures vivant sur Terre de façon minimale. D'après nous, la gravitation physique n'est pas le terrain où opèrent les causalités de l'astrologie. En fait, quelqu'un a calculé que le corps d'un médecin ou de la sage-femme exerce plus de force de gravité sur l'enfant que n'importe quelle planète distante. En revanche, la gravitation subtile et causale des neuf planètes influence la manière dont nous faisons l'expérience des résultats de nos actions, en contrôlant et en s'emparant de nos corps. Par analogie, bien que la pensée ait un minimum de réalité dans l'univers physique, elle est la cause de la plupart des actions physiques accomplies par les humains. »

DeFouw et Svoboda 1996: 34, notre traduction.

Le calcul astrologique consiste essentiellement à mesurer les trajectoires, les angles et les portées des astres ainsi que la manière dont les éléments (corps vivants ou non) qui peuplent la Terre sont touchés ou affectés par ce grand ballet. L'image d'une chorégraphie capricieuse d'influences «longue distance» s'infiltrant au plus profond des corps pousse chaque jour des milliers d'Indiens à nouer des relations souvent addictives avec les astrologues les plus divers. L'astrologie indienne possède ses cursus universitaires et génère des recherches très poussées pour expliquer des phénomènes aussi divers que les mouvements boursiers ou encore les grandes pathologies de notre temps. En se fondant sur de nombreux supports prédictifs que l'anthropologie commence à peine à explorer, les astrologues se donnent la possibilité d'établir, sans restriction d'objet, I'horoscope d'à peu près toute chose se trouvant sur la Terre (humain, animal, temple, maison, projet, etc. ${ }^{1}$ ). Cet élargissement considérable des techniques de divination ${ }^{2}$ soulève des questions qui méritent d'être explorées: comment de nouveaux systèmes de mise en résonance des astres sont-ils mis à l'épreuve? Comment qualifier les expériences auxquelles se livrent les astrologues? Et peut-on considérer qu'on a affaire avec l'astrologie à une forme «expérimentale» de savoir?

D'une consultation à l'autre, les connexions aux astres se cultivent, les astrologues font exister la matrice astrale un peu à la manière d'un environnement virtuel offrant de multiples possibilités d'action, un terrain de jeu dont le client est le personnage principal pour qui l'astrologue dégage de nouvelles possibilités de choix, sachant que cette matrice n'est rien d'autre que sa propre vie. En intégrant à ses diagnostics tout un lot d'entités à comportement ${ }^{3}$ qui agissent à distance et qui peuvent s'associer aux composantes du milieu selon des modalités qu'il revient au spécialiste de calculer et de commenter, l'astrologie indienne oblige à redéfinir ce que les Anglo-Saxons appellent l'interactive decision theory ${ }^{4}$. Que signifie vivre dans un monde où les astres sont reconnus comme des partenaires à part entière avec lesquels il faut composer, à la fois des liables, des liants et
1. Pour illustrer la dimension "non anthropomorphique» de l'astrologie en Inde, Caterina Guenzi donne l'exemple suivant: "Ainsi, la date de proclamation de l'Indépendance de I'Inde a été décidée après la consultation de plusieurs pandits astrologues et la nation indienne dispose d'un horoscope calculé sur la base de cette date. Sous l'ascendant (lagna) Taureau et le signe lunaire (rasi) Capricorne, le thème de la nativité de la nation indienne permet aux astrologues de fournir des interprétations et des prédictions à propos des relations avec le Pakistan, des résultats des élections ou de la victoire nationale de cricket. " (Guenzi 2013: 164-165)

2. Sur l'analyse pragmatique des consultations astrologiques en Inde, voir les travaux de Judith Pugh, sur l'astrologie à Bénarès, ceux de Caterina Guenzi et sur l'astrologie des temples au Kérala, ceux de Gilles Tarabout. Sur la variété des supports prédictifs de l'astrologie tamoule (des traces de pas au vol des oiseaux), voir la thèse d'Alexis Avdeeff (2014).

$$
\begin{aligned}
& \text { ci-contre } \\
& \text { fig. } 1 \\
& \text { Frontispice «Vision } \\
& \text { du monde angélique» } \\
& \text { in Daniel Defoe, Réflexions } \\
& \text { sérieuses et importantes } \\
& \text { de Robinson Crusoé faites } \\
& \text { pendant les avantures } \\
& \text { surprenantes de sa Vie. } \\
& \text { Avec sa vision du monde } \\
& \text { angélique, tome III. } \\
& \text { Amsterdam, L'Honoré } \\
& \text { et Chatelain, 1721. Coll. } \\
& \text { Universitätsbibliothek } \\
& \text { Erlangen-Nürnberg. }
\end{aligned}
$$

3. Ce terme me paraît plus juste ici que celui d'agent dans la mesure où il invite à être plus attentif aux propriétés spécifiques et pas forcément anthropomorphiques des entités en question.

4. L'autre nom de la théorie des jeux et de la modélisation (souvent sous forme d'algorithmes) de la manière dont les gens font des choix et optimisent leurs décisions. Voir Von Neumann et Morgernstern 1944 et pour une vision plus récente de la théorie des jeux Dutta 1999. À ma connaissance, à l'exception des travaux de David Zeytlin (1990,

1993 et 2012) qui abordent la question du calcul en divination, la complexité des calculs astrologiques n'a pas encore fait véritablement l'objet d'études anthropologiques. 
5. L'astrologie, l'astronomie et la physique ont servi de cadre d'invention à un grand nombre de pratiques de divination hybrides et parfois inclassables. Voir par exemple le travail d'Anthony Grafton sur Jérôme Cardan à la Renaissance (Grafton 1994 ; Cardan 2010 [1558] ; et aussi Curry [dir.] 1987).

Sur l'histoire du grand partage entre astrologie et astronomie, voir Simon 1992 ; Drévillon 1996 ; et dans le cas de la discipline astrale indienne, Pingree 1965; Minkowski 2002 : Guenzi 2013.

6. À titre d'exemple pour illustrer la multitude des associations possibles proposées par l'astrologie indienne, nous renvoyons à la carte proposée à la fin de notre ouvrage (Grimaud 2014). Elle a été réalisée à partir des informations données par le pandit Kulkarni auquel cet article est consacré. des lieurs au sens de Giordano Bruno (2001 [1591])? Sous l'étiquette d'astrologie, les praticiens n'ont jamais cessé d'explorer de nouvelles techniques, de tenter d'autres formes de calcul, de s'autoriser des correspondances inédites, mettant parfois même en place des dispositifs de consultation tellement singuliers qu'ils en deviennent difficilement catégorisables ${ }^{5}$. Si l'éther est bel et bien peuplé de forces et d'énergies en tout genre, comment déduire leur présence et les ressentir, si ce n'est en inventant de nouveaux dispositifs de capture et de lecture à leur mesure? Et si l'univers dans lequel nous sommes immergés agit comme une caisse de résonance qui ne cesse de véhiculer des ondes, des vibrations ou des radiations fluctuantes, comment les discriminer et les qualifier dans leur gradation d'intensité?

Les influences qui préoccupent les astrologues débordent et s'infiltrent partout: dans le corps, dans la psychologie des individus, dans les familles, les cuisines, la politique, en amour ou encore en économie. II faut distinguer ici entre les ondes de courte portée, mesurables à l'aide d'instruments (téléphones portables, etc.), et d'autres que l'on peut inférer (on en ressent les effets), mais non mesurer. Pour être en harmonie avec le monde qui nous entoure, juger du pouvoir bénéfique de telle ou telle action ou du poison que pourrait constituer telle ou telle relation, il faudrait s'en référer à ces influences d'une manière ou d'une autre et guetter leurs signaux. Les astrologues seraient un peu moins dépourvus que le commun des mortels devant ce que l'on pourrait appeler les causalités de «longue portée». Pour eux, il ne fait aucun doute qu'il existe d'autres forces, d'autres types d'emprise que la gravitation physique. Nous baignerions dans un champ énergétique d'emprises inconnues, à la fois physiques et mentales, qui influencent nos actions. Idéalement, l'astrologue devrait pouvoir, à partir de n'importe quel récit, objet ou corps composé, de la plante la plus rare à la boisson la plus chimique, remonter à ce qui magnétise ses constituants ou au contraire les désactive d'un point de vue astral, et ainsi tracer un faisceau d'influences (positives/négatives/neutres). Mais la question de savoir dans quelle mesure les emprises de «longue portée» qui font l'objet de l'astrologie sont mesurables ou non est souvent mal posée. C'est surtout au client de constater si les propositions que lui fait son astrologue entrent ou non en résonance avec sa propre vie. C'est son propre devenir qui constitue le terrain de l'expérience astrologique, le lieu même où les résonances se confirment ou s'infirment.

Cela fait-il de l'astrologie un savoir expérimental? Je crois que oui. Spéculatif certes, mais qui n'en reste pas moins profondément singulier quand on réalise la marge d'exploration qui est la sienne, entre sciences, médecine, éthique et divination: elle opère en effet un croisement unique entre les mathématiques, des formes particulièrement généreuses et bouillonnantes de relationnisme ${ }^{6}$ et les méthodes du mieux vivre, car c'est toujours à des fins d'amélioration ou d'optimisation que l'on vient consulter un astrologue. Même sans véritables instruments de mesure, les astrologues ne démissionnent pas devant les «immensurables». II faut bien leur reconnaître cette vertu. Mais, pour cela, ils ont besoin de la participation active de leurs clients qui vont eux-mêmes évaluer les effets positifs ou négatifs des liaisons qui leur sont proposées. Toute consultation divinatoire débouche donc, du point de vue du client, sur une série inévitable 


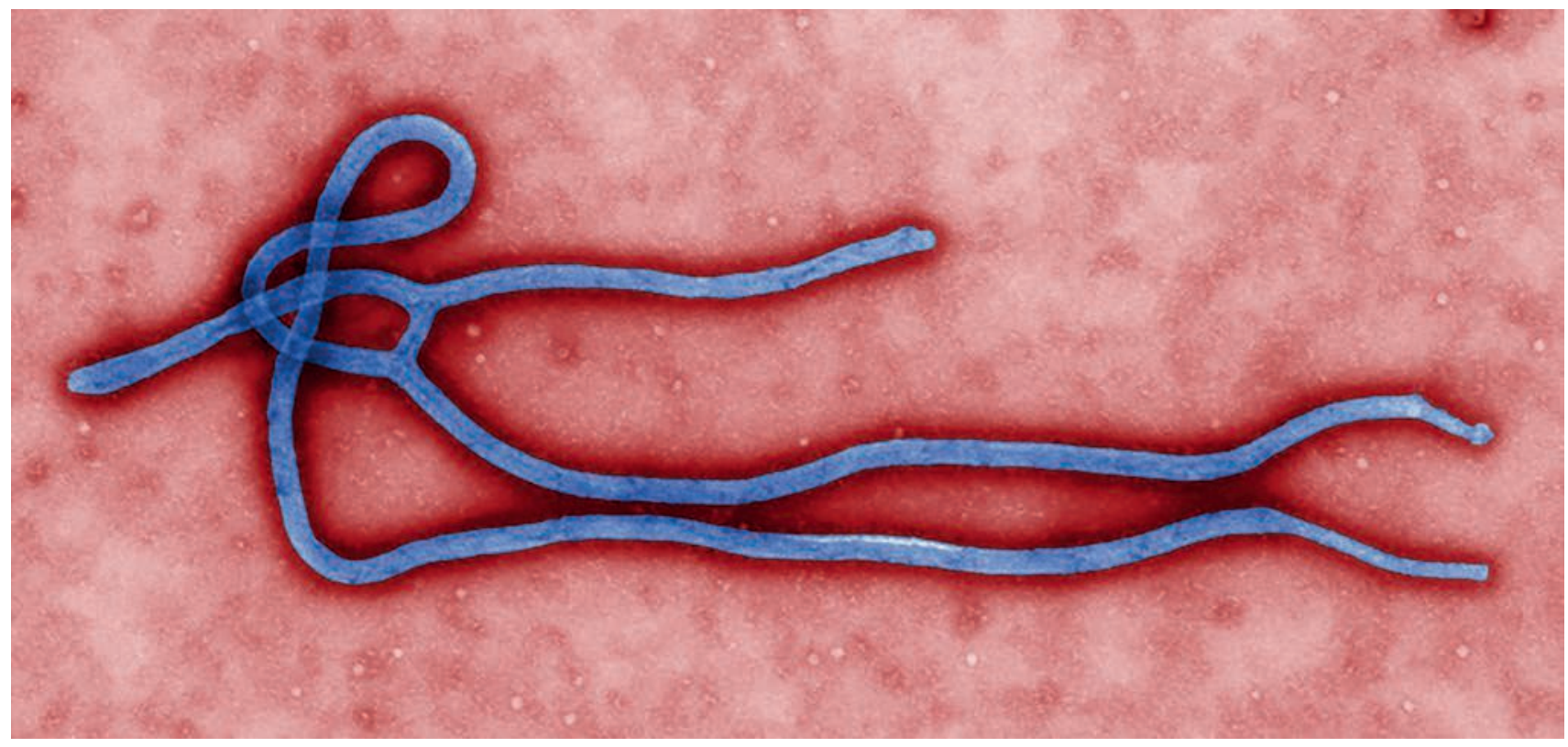

d'expériences et de tests qui se prolongent hors de la consultation et visent à éprouver dans son propre devenir des «cosmo-connexions». D'un côté, l'astrologue pratique une forme bien particulière de balistique, il ne convoque aucun missile ni canon, mais des entités dont il doit évaluer les trajectoires, les intensités de portée et les angles morts. De l'autre, le client qui se trouve lui-même engagé dans la matrice astrale est à la fois co-expérimentateur et expérimenté.

L'astromorphologie d'un pandit indien, le Dr Kulkarni, praticien d'une forme de divination par le trait, va nous permettre de donner un peu plus de consistance à cette idée: le cabinet d'un astrologue est le lieu d'expériences en résonance avant même toute supposition de causalité (Renou 1925). Le dispositif astrologique suppose à la fois une répartition d'éléments hétérogènes (la carte astrale), un acte de tuning (ou réglage), car ces éléments disparates n'apparaissent en résonance qu'à une certaine fréquence (celle des astres), et une forme de magnétisation: imprégnées par les astres, les composantes de l'environnement se dotent de nouvelles tonalités et entrent en réverbération d'une certaine façon, sous certaines modalités qu'il revient au client de juger. Comme nous allons le voir, le pandit a intégré à la panoplie d'outils de l'astrologue le tracé, une nouvelle manière de convoquer et de faire parler les astres sous la forme de traits et/ou d'ondes.

\section{Traits, points et couches}

Fils d'astrologue, le pandit Kulkarni a travaillé de longues années comme dessinateur sur des chantiers de fouilles archéologiques et a peu à peu acquis sa renommée en faisant des portraits d'individus, sans les avoir vus, à partir de leur horoscope. Les centaines de portraits qu'il a laissés constituent les traces saillantes d'une recherche plastique, aux frontières de l'archéologie, de l'anthropologie physique, de l'art et de la divination. Les individus lui ayant servi de modèle ont fini par former au fil des années une sorte de matériau unique par son ampleur pour éprouver sa technique fig. 2

Virus ebola. Des astrologues indiens y ont vu l'influence de Rahu et Ketu dont les formes graphiques sont les suivantes:

\section{८ ช Rahu Ketu}


7. Outre l'article de Binet (1887), on pourra consulter les pages qu'Andreas Mayer consacre à ce précurseur de la psychologie expérimentale et aux techniques qu'il utilise pour «substantialiser les hallucinations " (Mayer 2013).

\section{ci-contre}

fig. 3

Univers en expansion. Dessin. Rajasthan, $\mathrm{xVIII}{ }^{\mathrm{e}}$ siècle in Ajit

Mookerjee, Tantra Asana. A way to selfrealization. Basel-ParisNew Delhi, Ravi Kumar, Basilius Presse, 1971 planche 8 p. 23 de dessin. Le pandit est devenu probablement l'un des plus grands portraitistes encyclopédiques que l'Inde moderne ait jamais connu, mais son entreprise semble avoir été largement ignorée en dehors des cercles astrologiques. Ses clients l'ont préféré à tout autre astrologue car il était unique et recourait à un genre d'astromorphologie qu'il était le seul à pratiquer dans la ville où il résidait. II leur offrait par sa technique du portraitrobot un diagnostic plus incarné que celui de ses collègues, suivant, à même leur visage, les possibilités de mesure des emprises astrales et de leurs portées graduées.

Pour saisir ce qui se passe dans le cabinet de cet astrologue, il faut revenir à une intuition d'Alfred Binet, ce précurseur français de la psychologie expérimentale de la fin du $x x^{e}$ siècle qui se mua, avec son collègue Charles Ferré, en "chasseur d'hallucinations" dans un travail passionnant sur les images mentales ${ }^{7}$ (Binet 1887). La question à propos des images mentales, nous dit-il, est moins de savoir si elles sont vraies ou fausses que de mesurer leur intensité et leurs modes gradués d'émergence. «ll faut s'habituer à considérer une image comme pouvant passer par les mêmes degrés d'intensité qu'une contraction musculaire", écrit-il. II ajoute: "Cette qualité de l'intensité est généralement négligée en pratique, car ce que nous recherchons dans les images, c'est une qualité tout à fait différente et indépendante de la première, c'est-à-dire la vérité. Mais la vérité n'est rien sans l'intensité. Quand deux raisonnements sont inégalement forts, c'est le plus fort qui triomphera, qu'il soit vrai ou qu'il soit faux. On ne parle pas de vérité en mécanique; il n'y a que les forces qui agissent; il en est de même en psychologie, toute discussion, toute délibération est au fond un problème de cinématique. En étudiant l'intensité des images, nous étudions la manière dont, en fait, se fondent nos convictions, vraies ou fausses.» (Ibid.: 473) À l'appui de son argument sur l'intensité des images, Binet convoque les expériences réalisées sur les hallucinations qui selon lui sont localisables dans la réalité, traçables grâce à toutes sortes d'appareils et correspondant à des points de repère très précis dans l'environnement où elles se produisent. II s'intéresse par ailleurs à la suggestion (dans l'hypnotisme), où «l'intensité de l'image suggérée est en quelque sorte en rapport avec l'intensité de l'impression suggestive, parole ou geste» et où «l'association d'idées devient une véritable ligne de force" que l'on peut comparer au "fil métallique qui transmet la force d'un moteur magnéto-électrique» (ibid.). La technique du pandit qui consiste à dessiner le visage de ses clients et à déceler à même les traits du visage les influences des astres joue de ressorts tout à fait comparables à ceux étudiés par Binet. Elle suppose une méthode savante pour identifier des "points de repère" sur le visage. Le portrait astral est ensuite précisé progressivement à partir de ces points et la personne, en voyant ainsi son portrait composé de lignes de force "activées", achève alors son astromorphose (ou intériorisation de sa situation astrale) en contemplant ce miroir cosmographique d'elle-même. Dans ce contexte, le portrait astral est-il une simple représentation du jeu des forces cosmiques? Ou implique-t-il une forme de transmission magnétoélectrique (au sens de Binet) qui ferait de l'astrologue l'agent d'une traduction figurative des ondes astrales? Ou bien faut-il imaginer un autre type d'enchaînement, mêlant les deux, où les images seraient douées d'une force de réverbération qui leur serait propre? 


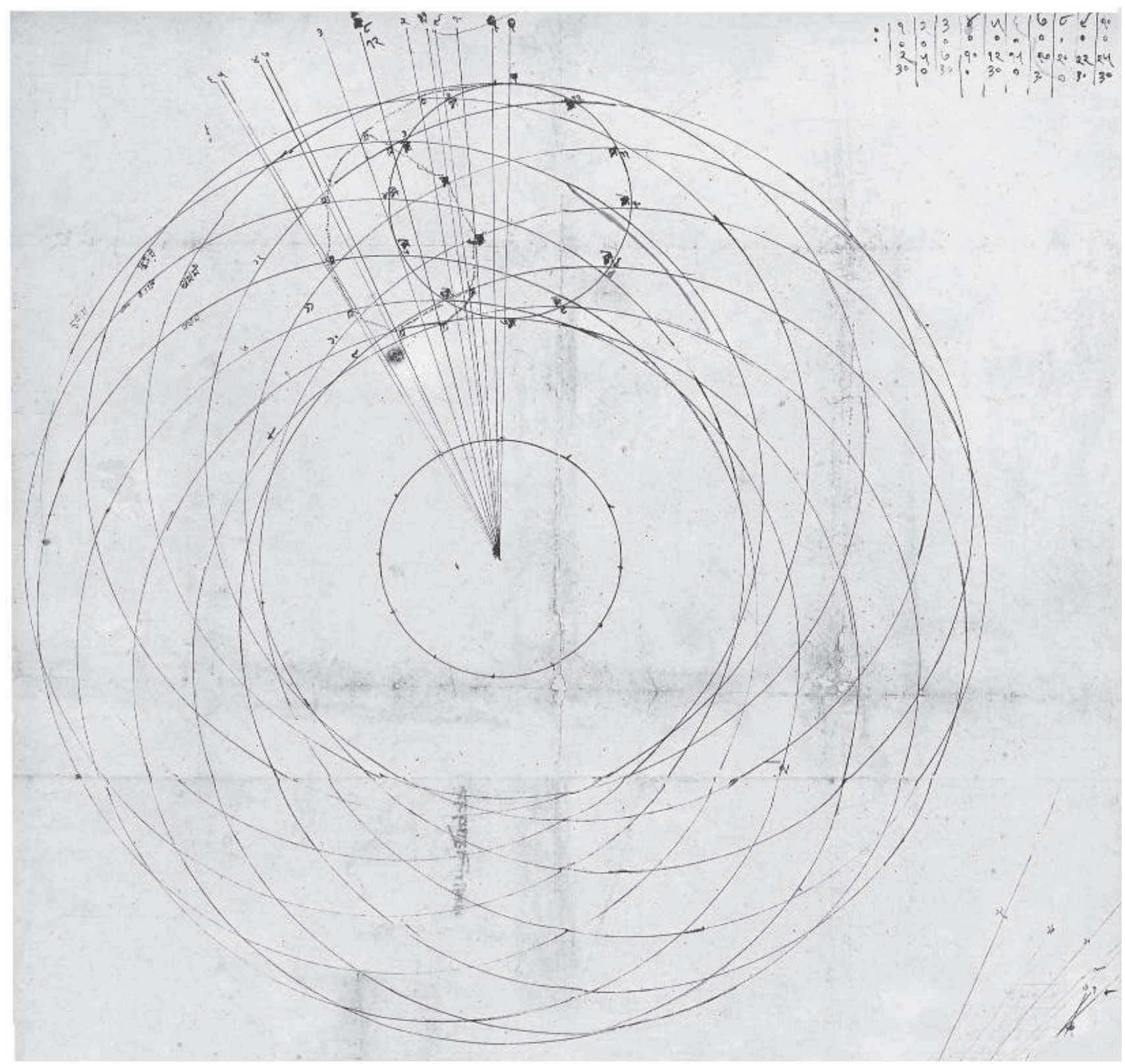


Les transports d'ondes sont généralement classés en diverses catégories (onde oscillante, solitaire, de choc, électromagnétique, acoustique, etc.). Qu'en est-il ici? Durant la consultation, les influences des planètes sont toujours précisées par l'astrologue: les astres entretiennent entre eux et avec les Terriens des rapports d'attraction et de répulsion, d'excitation, d'apaisement, de rayonnement, d'affaiblissement, de tension, de perturbation ou de neutralisation. Ces changements d'état, de même que la possibilité de qualifier les degrés de force ou d'intensité (bal) de telle ou telle emprise (prabhav) planétaire particulièrement bien placée, sont essentiels à la balistique astrologique et font l'objet d'une interprétation. II revient à l'astrologue de déterminer ensuite comment l'individu est affecté, quelles parties de son corps pourraient être touchées, les angles morts laissés par les planètes, les phases favorables ou défavorables, ainsi que le soutien que pourrait lui apporter telle ou telle entité. Mais rien dans la boîte à outils de l'astrologue ordinaire ne permet de projeter ces différences d'intensité sur le papier. Or Kulkarni, avec le portrait astral, a tenté de traduire ces influences en une gamme de traits, déplaçant le problème de l'onde astrale dans un cadre pictural. Avant de voir comment il s'y prend, disons quelques mots des métaphores auxquelles il a recours quand il parle de son travail car elles permettront de mieux comprendre en quoi le cosmos des astrologues n'est pas tout à fait le même que d'autres cosmos.

C'est comme si les vagues que je ressens dans mon cerveau contenaient tous les visages possibles. Et ce n'est que lorsque l'image est pleinement détaillée dans le cerveau que mon étude peut commencer. Quand j'ai une telle image en tête, je la dessine sur le papier. Quand je ferme les yeux et que j'écoute l'histoire de mes patients, cette histoire se projette sur mon écran intérieur. Elle se matérialise et je commence à dessiner. Les mots deviennent des images. Le cerveau est la meilleure caméra que l'on puisse imaginer. Et le mien en particulier. Je n'ai pas besoin de réfléchir bien longtemps ou de me concentrer pour voir surgir les images. Entre mon cerveau et ma main, la connexion est très fluide. C'est du très haut débit, 500000 mégabits! Mon travail est machinal. Mon cerveau est devenu une sorte de bibliothèque où sont entreposés des milliers d'horoscopes. À moins que ce ne soit une usine...

Habité par une sorte de "cinématographe intérieur", se présentant volontiers comme une "usine à produire des horoscopes", Kulkarni a répété tant de fois le même exercice de visualisation qu'il se sent machiné, mais machiné par qui ou par quoi? Certes, on peut voir dans ce besoin de se mettre en scène à tout prix comme un appareil une façon d'objectiver la prétention statistique et mathématique de l'astrologie, la mécanicité à laquelle elle aspire, mais je pense qu'on peut y voir aussi l'illustration magistrale d'un désir de connexion tous azimuts. L'astrologue est à la fois interprète et instrument. II fait parler le cosmos autant que le cosmos le fait parler et même peindre. La matrice astrale ou le "grand scanner cosmique ", comme il se plaît à nommer le bain de radiations dans lequel nous vivons, serait alors comparable à une sorte de générateur énergétique pourvu d'autant de «têtes" qu'il existe de planètes, l'astrologue en constituant la main attentive, une sorte d'extension prothétique. 


\section{Tracé de l'onde astrale et interactivité}

Regardons de plus près comment le pandit s'y prend pour faire un portrait astral et comment il se sert conjointement de celui-ci et de l'horoscope pour renforcer ses prédictions. Quand un consultant se rend dans son cabinet, l'astrologue est attentif à tous les détails qui lui échappent et participent de son diagnostic, ou en tout cas l'influencent dans les questions qu'il pose.

Pourquoi votre nez est-il un peu décalé vers la gauche? Pourquoi vos oreilles sont-elles plus longues que les miennes? Et votre front plus long et plat que le mien? Et pourquoi avez-vous une narine plus ouverte que l'autre? Si vous venez me voir, je décèle tout ce qui vous échappe et que vous ne voyez plus, car personne ne peut se voir tel qu'il est, même dans un miroir. II ne rencontre que son image inversée.

Le pandit travaille à partir de ce qui se voit «à l'insu du sujet ». II relève un certain nombre de points et de lignes, dont on ne peut malheureusement ici que donner quelques exemples (fig. 5 et 7). Alors que je cherchais à mieux saisir ses sources, il m'a dit que je pouvais aller voir dans les textes, mais que l'interprétation physiognomonique était incorporée à son œil capable de «relever les détails tel un appareil photo surpuissant ». J'ai d'abord souri et n'ai pas compris immédiatement ce qu'il voulait dire. J'ai cru pendant longtemps qu'il pensait avoir dans sa peinture l'exactitude d'un appareil photo, et que celle-ci révélait une sorte de «super capacité » à voir les détails. Mais, en réalité, il dit bien autre chose. II va de soi qu'aucun traité d'astrologie ne fait référence à l'informatique ou à la photographie. Si l'astrologue ne cesse de se comparer soit à un ordinateur soit à un appareil photo, ce n'est pas parce qu'il considère avoir l'exactitude d'un appareil photo, mais plutôt pour la raison suivante: de même que son œil, par habitude, «va aux détails pertinents ", comme il le souligne, ses portraits captent l'essentiel de la personne, soulignant les traits pertinents qui échappent à sa propre perception, un peu comme une photo qui, par son surplus de détails, nous donne une image forcément décalée de la perception que nous avons de nous-même.

Si le pandit aime les métaphores technologiques afin d'appuyer la scientificité et l'exactitude de ses procédés, l'observation des consultations donne une autre image de sa technique, moins mécanique (ou mécaniste) et peut-être plus convaincante parce que plus interactive. II existe une différence entre les «portraits de travail» conservés par le pandit et ceux qu'il propose à ses clients, dessinés au cours de la consultation. Les portraits destinés aux clients épousent tous le même format, le visage au recto et le crâne en surimposition au verso de la page. Les portraits de travail conservés par l'astrologue affichent le plus souvent le crâne et le visage sur la même page et ils sont généralement accompagnés de commentaires à caractère personnel. Le pandit les a écrits pour lui-même, afin de progresser dans sa propre recherche, et ils sont très souvent d'une grande poésie. On n'en donnera que quelques exemples. À propos d'une femme jouissant d'une «bonne réputation dans les milieux intellectuels», il écrit:

La Fortune, Jupiter et Vénus sont en haute position au quadrant nordouest. En conséquence, elle n'obtiendra le bien-être qu'après 24 ans. 
fig. 4

"Serpent. Carte des étoiles ", in Johann Bayer, Uranometria Augustae Vindelicorum Excudit Christophorus Mangus. Représentations des 48 constellations ptolémaïques sous forme de carte dans un atlas.

Coll. Linda Hall Library, LHL Digital Collections.

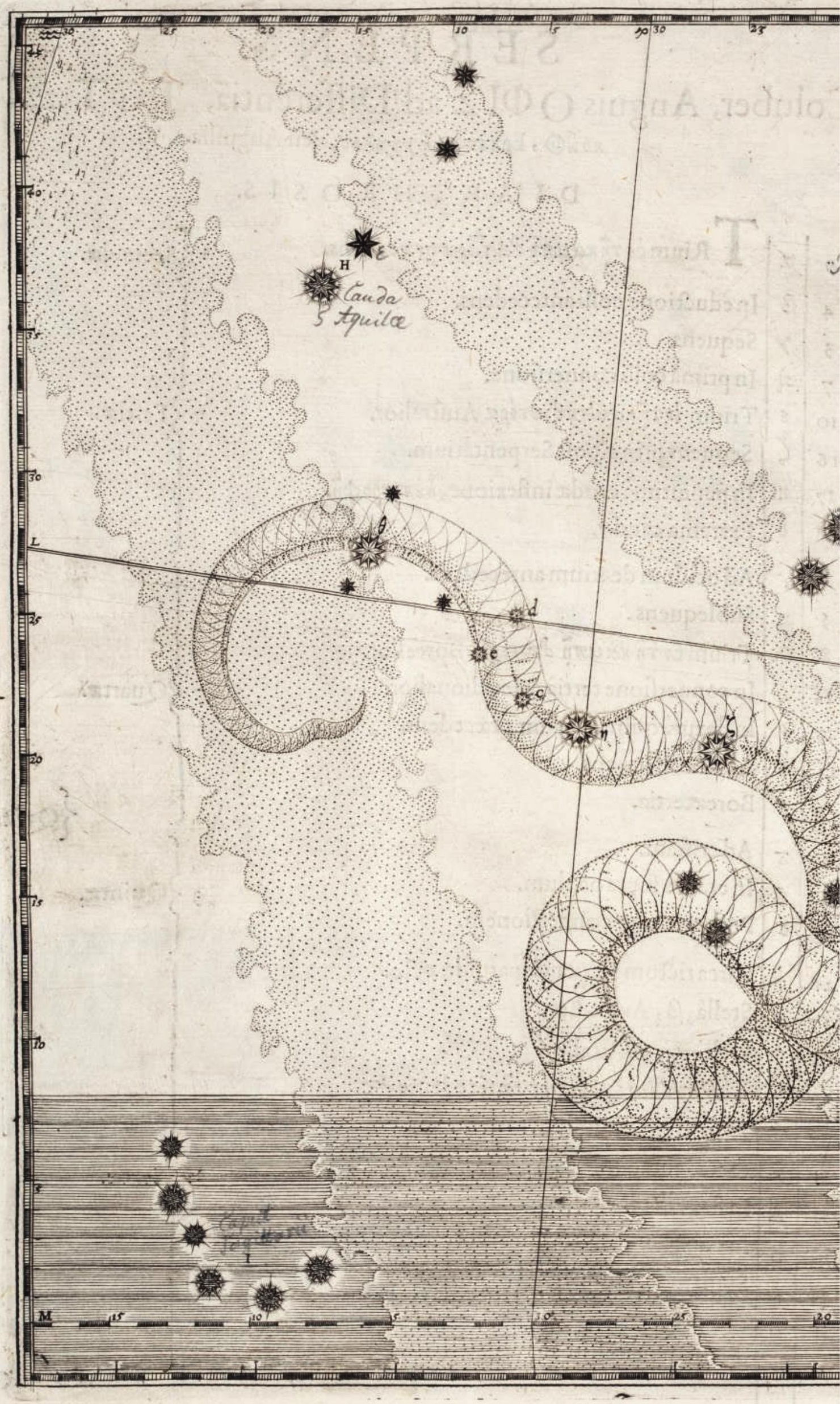




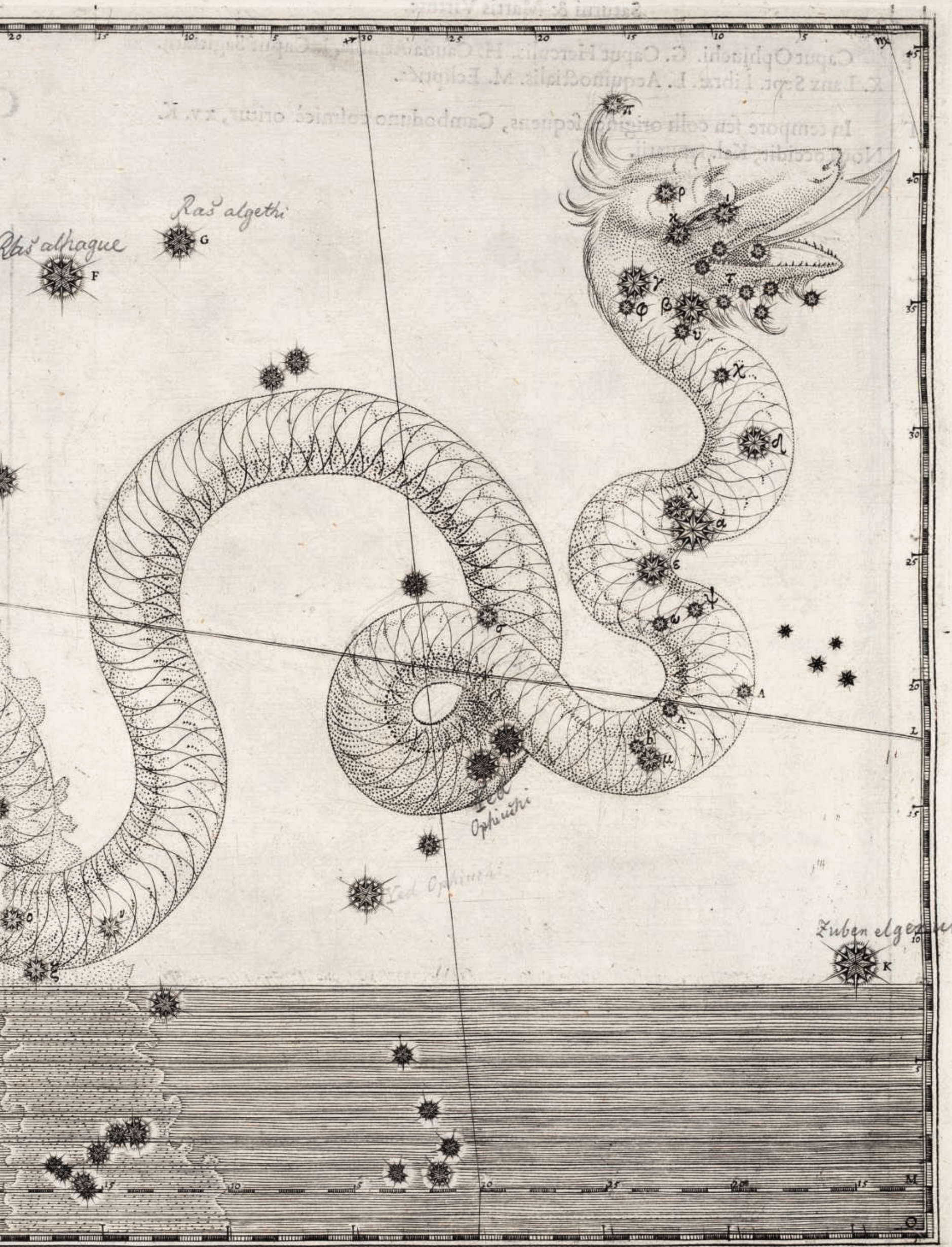


Après juin 2002, Rahu sera en Gémeaux et détruira l'ennemi complètement et Saturne en Poissons achèvera de vaincre son ennemi... À cause de l'influence de Jupiter et de Vénus, elle bénéficie d'une beauté rare, d'une figure et d'un corps magnifique. Ketu en Poissons lui donne ses cheveux denses et ses grands yeux. La position du dieu de l'Éducation et de celui de l'Ennemi influe sur la Connaissance. Celle du Mari est alignée sur celle de la Connaissance dans l'horoscope. Elle se mariera vraisemblablement avec quelqu'un qu'elle rencontrera sur son lieu de travail. Son patron sera aussi celui de son mari. Cependant, avant cela, elle commettra une erreur sérieuse et voudra se marier pour y remédier. Mais, entre-temps, elle aura été flouée car l'ombre de la Mort se profilera déjà sur son corps.

Un homme recherché par la police donne lieu au portrait suivant:

Homme en état de fuite, date 28-10-1993. Le dieu de la Mort, Vénus, est en face du dieu de l'Intelligence et il sort en Vierge placée en basse position. Du fait de cette configuration, son esprit s'est rempli d'un désir d'émancipation mais ses ennemis se sont emparés de son corps.

À propos d'une femme connue pour attiser la passion des hommes:

Date de naissance, 19 novembre 1977, aube, 6 h 15. Horoscope de naissance et de mariage: beauté arrogante. Femme prétentieuse, dotée d'yeux capables d'éveiller les passions masculines violentes. La Nature n'a pu donner naissance à une telle créature que dans un moment de distraction. Le Corps est influencé par le Soleil et Budh, I'Intelligence, influence son visage, la souplesse de ses traits et sa fragile harmonie. D'où aussi son teint de lait brillant au pouvoir magnétique, ses os forts et la constitution solide de ses muscles influencés par Budh. La beauté de Vénus est due à l'influence de la Balance.

Et, au dos du portrait d'une jeune fille en âge de se marier:

Visage serein. Les yeux reflètent le sens de l'hospitalité, l'amour, l'affection de la bonne famille dans laquelle elle est née. Saturne a rencontré Jupiter en "Bhagyaswami» et «Mokshaswami», elle a perdu le contentement et sa nature, son attitude et sa religion ont changé. Elle est perdue dans ses pensées. «Ne m'épouse pas sans mon consentement!»

Insistons sur le fait que ces commentaires sont des ébauches, des tentatives de description ou des hypothèses, tout comme le carnet de notes de l'artiste ou du chercheur enregistre les tâtonnements associés à une technique ou à une méthode. Loin d'être à sens unique (de l'horoscope connu vers le visage inconnu), la technique du portrait-robot astral doit donc être comprise comme un jeu «multipolaire» à trois termes (horoscope, crâne, visage) dont l'inconnue peut varier et se porter sur un ou deux de ces termes. Pour peaufiner son art, le pandit s'est inspiré par exemple des visages déjà connus de personnages historiques dont on ignorait I'horoscope. II a aussi reconstitué des visages (associés à des horoscopes 


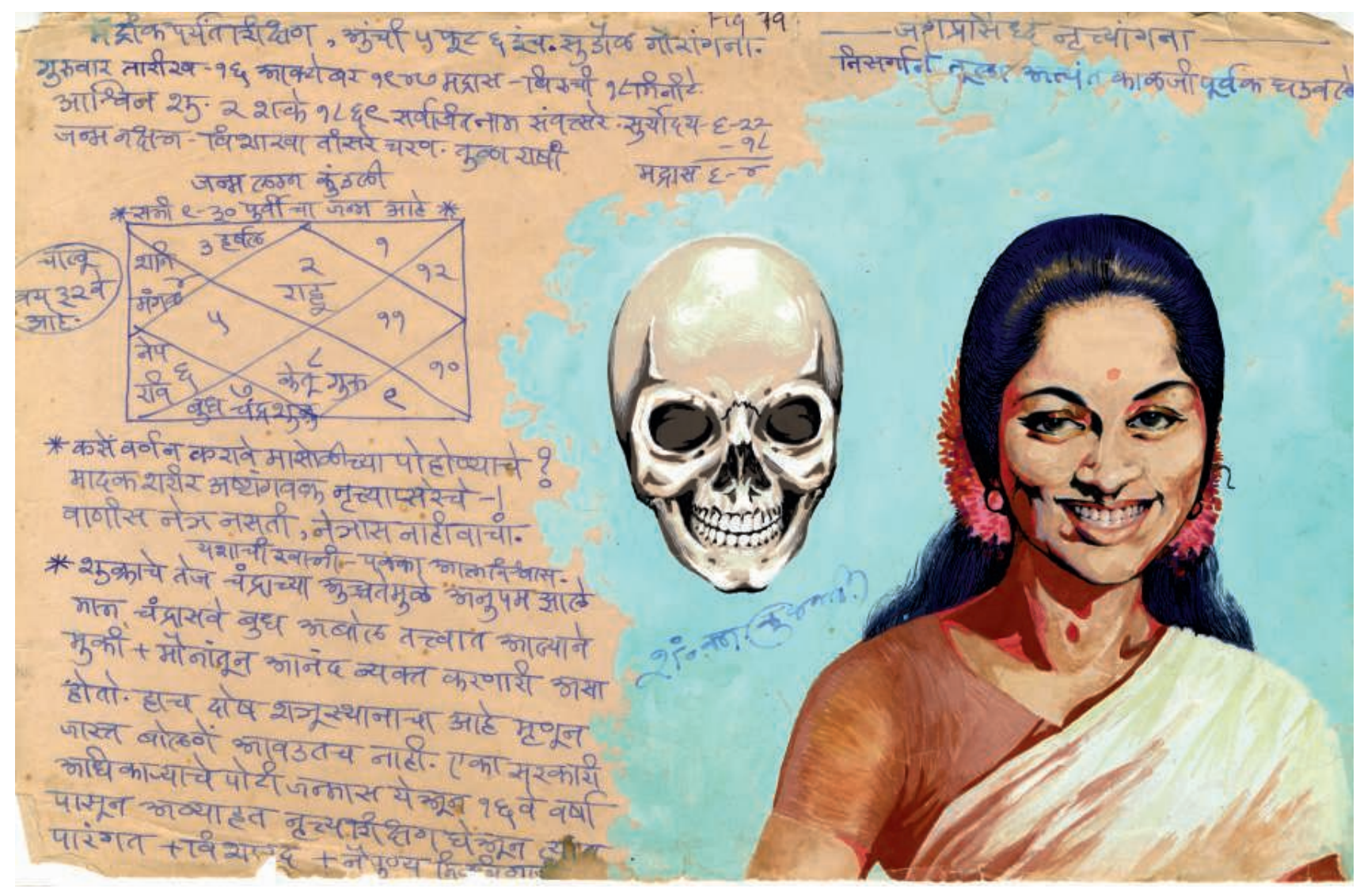

fig. 5

Portrait dessiné et annoté par Kulkarni.

Les annotations traduites par Archana Bharate et Emmanuel Grimaud signifient:

Comment décrire les mouvements d'un poisson dans l'eau? II est aussi difficile de définir le corps féminin de cette nymphe lorsqu'elle danse! Les mots sont sans pouvoir et les yeux ne peuvent parler! Son succès est confirmé - elle a une forte confiance en elle. La brillance de Vénus est due à la position haute de la Lune. Mais Mercure étant taciturne, elle est réservée et exprime son contentement et ses sentiments discrètement. En conséquence, elle n'aime pas bavarder. C'est la fille d'un bureaucrate du gouvernement. À l'âge de 16 ans, elle prendra des leçons de danse et acquerra une grande maitrise de son art.

Danseuse célèbre, bien éduquée, taille: 1,70 mètres. Beautée au visage clair. Date de naissance: jeudi 16 oct. 1947 - Madras, Biruchi Ashwin Vishakha $3^{\mathrm{e}}$ quadrant Balance. 
possibles) à partir de crânes archéologiques. Ce n'est qu'en déplaçant ainsi l'inconnue sur l'un ou l'autre des pôles orientant son interprétation conjecturale qu'il a pu affiner sa méthode.

Notons que les motifs qui poussent les gens à consulter le pandit sont variés. Lorsqu'il faut expliquer un événement, comme la mort de quelqu'un, le pandit se prononce uniquement à partir de l'horoscope. Et si le consultant lui demande de dresser son portrait, il le fait, mais celui-ci ne sert pas forcément de support prédictif. D'autres viennent le consulter pour obtenir un diagnostic médical, résoudre des problèmes de fertilité, identifier des risques de maladies éventuelles. II se sert alors conjointement de l'horoscope et d'un examen du visage et du crâne. Mais, dans la plupart des cas, le portrait répond à un besoin plus global: la personne, en emportant son portrait, prolonge la séance du pandit. Elle peut se contempler chaque matin et intérioriser par imprégnations successives l'astromorphose de soi entamée lors de la consultation. Le pandit insiste ici sur la vertu apaisante de l'effet de miroir ainsi créé, le portrait astral devant progressivement se substituer, à force d'être perçu par son sujet, à l'image de soi.

L'horoscope reflète l'image de la personne comme un miroir. Cette image devient une forme ou une figure de la personne et cette forme se concrétise dans la réalité. Elle prend une apparence concrète. Cette apparition devient la sienne propre. Et c'est pourquoi l'horoscope de naissance peut être superposé. Expose cette image à l'émulsion du pouvoir de ta volonté et de ton désir! Et fais en sorte que l'invisible devienne visible!

Ajoutons à cela que le portrait opère une montée en présence des forces astrales en offrant une sorte d'image «paralysée» du sujet figé ici sous l'emprise de celles-ci. Il faut donc figurer au mieux le «danger de la personne ", le risque à la côtoyer ou à l'effleurer. Ainsi, le visage d'un gangster venu consulter Kulkarni lui inspire le commentaire suivant:

Un bon portrait est un portrait qui donne à sentir le danger que représente une personne et le risque que l'on court en l'approchant. Regardez donc le visage de ce marchand. II est effrayant et aussi dangereux qu'une bombe à hydrogène. Un homme explosif. Je connais de nombreux gangsters de ce pays, ils sont venus me consulter. J'ai conseillé beaucoup de bandits, non pas parce que j'approuve leurs actions, mais parce qu'ils viennent me voir. Ils ne feront rien sans consulter les astres. S'ils vont jusqu'au bout de leurs actes, c'est que ces actes devaient de toute façon se produire!

Le portrait matérialise donc les tendances et penchants propres à toute physiognomonie, mais ceux-ci sont ici évalués et réappropriés par le consultant dans un cadre plus large, la matrice astrale. À aucun moment, le pandit n'entre avec ses patients dans les motivations de leurs actes, il ne fait que constater leur inéluctabilité. Les Terriens sont mus par des passions de toutes sortes qui les dominent et sont les incarnations, en acte, des emprises plus profondes se manifestant à leur insu dans leur plasticité. Dans la balistique astrologique, l'emprise apparaît d'autant plus polymorphe 
qu'elle a plusieurs surfaces d'exercice: elle peut s'exercer d'une planète sur un crâne, d'un crâne sur un visage, de son propre visage sur ses actes. Le consultant peut, au bout de cette chaîne psychophysique de transmission qui inclut la représentation que le sujet se fait de lui-même, être effrayé par son propre portrait autant que l'astrologue est lui-même remué par les passions qu'il cherche à matérialiser. La balistique astrologique met le sujet face à des puissances à l'état pur, ainsi que l'astrologue qui se conçoit comme partie intégrante du dispositif d'expression, surtout quand il rend présent le courant inéluctable qui fait d'un homme «une bombe à hydrogène", l'instrument de forces qui s'expriment à son insu. La superposition du crâne et du visage est conçue par le pandit comme une opération de rééquilibrage, il «remet d'aplomb», il permet de voir ce qu'on ne voyait plus.

Il faut s'attarder sur cette vision de la personne comme étant constituée à la fois de «couches» et de «points» imperceptibles au sujet lui-même. Notre pandit engage son patient dans un exercice mathématique de redressement de son image et de ses coordonnées qui passe par l'examen de «ce qui lui échappe». La topographie du crâne devient dans le cabinet de l'astrologue une sorte de «sous-couche " cosmographique dans laquelle on peut lire. Mais est-ce au même titre que sur les lignes de la main ou sur le visage? De même qu'un bon dessin anatomique s'appuie sur celui du crâne, un bon portrait est «osseux», il doit laisser transparaître le squelette derrière les parties molles. Ce n'est qu'après avoir assisté à plusieurs consultations où le pandit palpait le crâne de son patient que j'ai réalisé à quel point l'attachement du visage au crâne a son importance dans son diagnostic. Ses portraits portent la marque de ces palpations diverses. À quoi l'astrologue a-t-il exactement accès par le crâne qu'il ne peut approcher que par le visage? «Le crâne est le négatif du visage et l'horoscope le négatif du crâne", dit-il. Tout cela forme à première vue un assemblage de couches d'impression cohérentes reliées entre elles sur un mode photographique jusqu'au visage qui en constitue le développement positif. Du point de vue du diagnostic, l'astrologue semble pouvoir davantage rentrer dans les détails des influences à partir d'un visage que d'un crâne, tandis que le crâne est tenu pour fournir une carte moins précise mais plus claire des influences. Quand on passe de l'un à l'autre, le diagnostic s'enrichit. Le crâne sert bien souvent dans la consultation à convoquer les "grands penchants" ou les «passions fortes», tandis que le visage permet à l'astrologue des jugements plus subtils et plus précis sur la psychologie de l'individu. En dissociant le crâne et le visage, les emprises s'affinent et se différencient. Le crâne et le visage sont organiquement liés par des tissus musculaires qui s'attachent à la boîte crânienne. L'emprise est ici organique et visible dans les parties molles du visage. C'est la prégnance des tissus qui marque l'emprise du crâne sur le visage. Mais les emprises des planètes sur le crâne, quant à elles, doivent être retracées patiemment, de manière mathématique, à partir de l'horoscope. Elles ne se voient pas, elles ne se «palpent » pas. II s'agit de rayons invisibles dont crânes et visages subissent les effets. La façon dont des actes, des passions ou des événements singuliers peuvent ensuite être liés à chacun de ces supports dépend de la consultation et du consultant. Selon les cas, l'astrologue va plutôt mobiliser l'un ou l'autre, les deux et le plus souvent les trois dans son interprétation. Voilà qui conduit à penser qu'en dissociant l'horoscope, le crâne et le visage comme trois négatifs, 


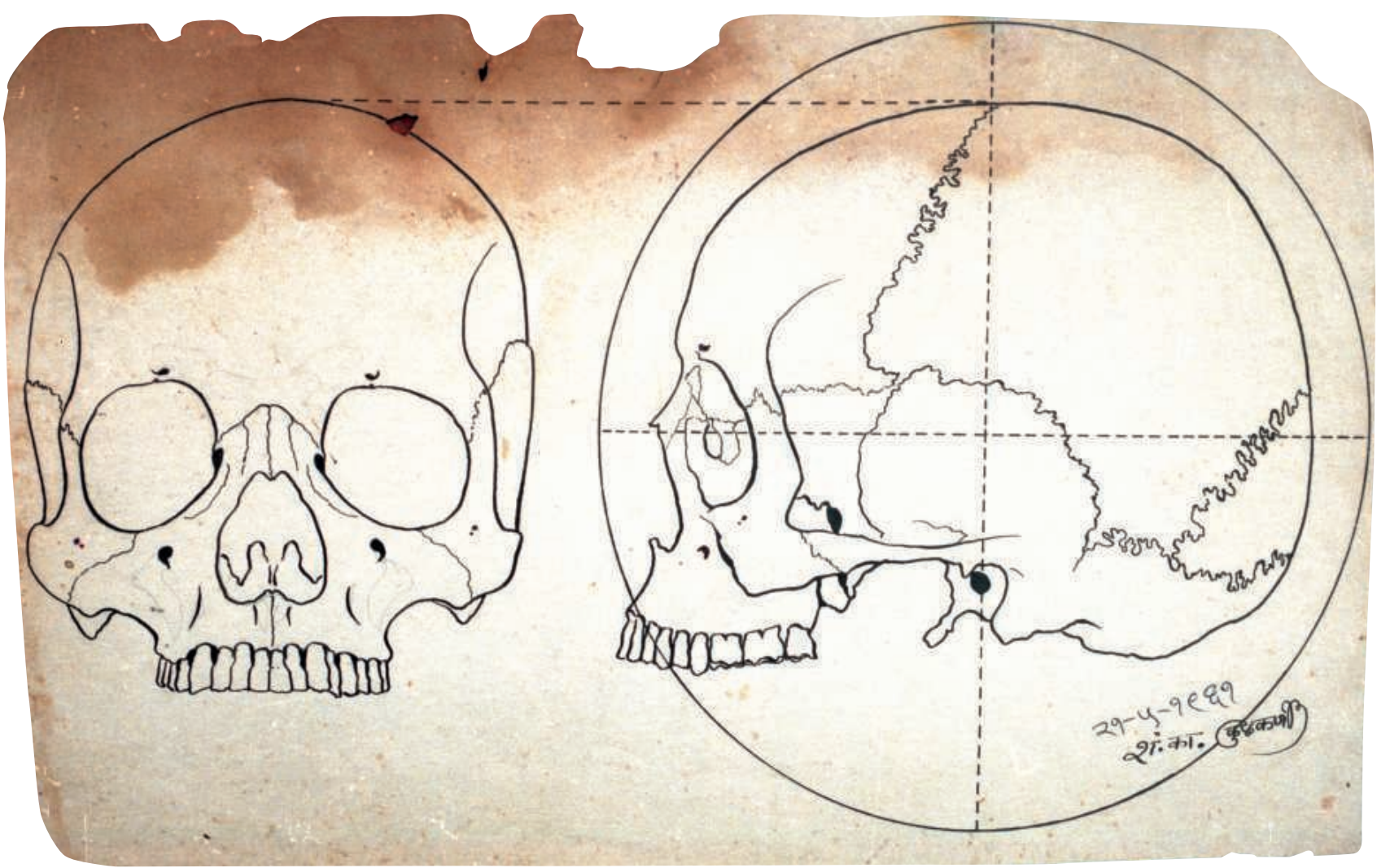

fig. 6

Projection sur crâne.

Dessin de Kulkarni. 
la logique d'attribution des causalités s'enrichit, fournissant à l'astrologue trois manières de produire du diagnostic au lieu d'une.

On mesure ici toute la différence entre cette logique permettant de fractionner les supports et de multipolariser l'interprétation astrologique et la phrénologie de Franz Joseph Gall ${ }^{8}$ (1810) qui semble uniformément «orientée», enfouissant les penchants et les passions à l'intérieur de la boîte crânienne pour qu'ils n'en sortent plus (si ce n'est par la voie de l'hérédité). Le pandit, à l'inverse, ne localise ces penchants que pour mieux les délocaliser dans le même élan, hors du cerveau. II a suffisamment à faire avec le crâne, le visage et les astres pour pouvoir se passer du cerveau qui n'est plus ici qu'un fantôme. Kulkarni disloque les «penchants» de la phrénologie issus des "profondeurs de l'âme humaine» (comme dirait Gall) pour produire des jeux de causalité à trois termes: le visage, le crâne et l'horoscope, tantôt dissociés, tantôt associés, permettent de rendre un diagnostic circonstancié sur la diversité des emprises issues des «profondeurs du cosmos». L'astrologie devient une balistique des passions. La physiognomonie ne vaut que parce qu'elle permet d'ajouter le visage comme point de chute à des mouvements orbitaux qui, lorsqu'ils rencontrent la matière vivante, «font» passion. Assurément, Gall aurait refusé ce tour de passe-passe virtuose et, avec lui, les connexions entre les crânes et les astres identifiées par le pandit; libre à chacun, en effet, de discréditer cette approche des ondes vibratoires en raison de son côté hybride et "patchwork». Celle-ci mêle les astres, un brin de physique, des hypothèses archéologiques sur l'évolution, des appréciations esthétiques sur les personnes et enfin des données de nature psychologique sur les comportements. Néanmoins, il faut saluer la prouesse qui vise à fractionner la personne et à l'ouvrir ainsi sur un champ de forces pour l'aider à mieux s'approprier son devenir. Certes, Kulkarni semble faire de la psychologie sans jamais passer par le cerveau, mais sa manière de décomposer et de connecter les comportements, les traits du visage et les astres est peut-être au fond porteuse d'un autre modèle de la personne, plus proche de ses oscillations discrètes et discontinues. Pour lui, le visage est toujours un carrefour de tensions conflictuelles. Ce qui fait la singularité d'un Terrien ne peut lui être dicté par lui-même, il n'est qu'un corps réceptif à des courants de toute nature, et il n'est particulier que parce qu'il est singulièrement affecté par ces courants. Toute la modernité de Kulkarni est là. À aucun moment il ne dissocie ces forces (sociales, familiales, astrales), il les considère toutes pour fournir un portrait et s'appuie tantôt sur le crâne, tantôt sur le visage pour soutenir son diagnostic. Si c'est le cosmos qui se dote d'une sensibilité (d'action) plus que le cerveau qui acquerrait une profondeur, le redressement cosmographique de soi s'opère de deux manières, ainsi qu'on l'a suggéré. L'astrologue fractionne d'abord le visage au millimètre près car l'activité des astres est lisible sous la forme de «points de contact» ou «points d'activation », invisibles au sujet mais matérialisés par le dessin. Les astres influent ensuite sur la personne par «couches d'impression», donnant lieu à toute une gamme de traits et de zones, des plus visibles aux plus profonds (expressions, parties molles, parties dures, etc.), que le dessin vient actualiser. Par le portrait, l'astrologue embraie son patient dans un mouvement centrifuge paradoxal: plus il cartographie de points et de lignes singulières, plus il le
8. Pour une histoire

de la phrénologie au XIX $x^{e}$ siècle, voir Renneville 2000. Pour un bel exemple de manuel de lecture des traits du visage fait pour le grand public, mais très surprenant par son imagerie, Vaught 1908 [1902]. 
9. Voir les chapitres que Caterina Guenzi consacre à l'inventaire précis de ces remèdes, qui varient selon les circonstances et les types d'astrologie (Guenzi 2013).

10. Sur l'importance du tracé comme catalyseur d'attention dans d'autres techniques de divination, notamment au bâton, voir par exemple Liberksi Bagnoud 2012.

\section{ci-contre}

fig. 7

Dessin de Spana, qui signifie "rêve", par Kulkarni. Annotations de Kulkarni traduites par Archana Bharate et Emmanuel Grimaud. Photomontage de Sophie Laporte. situe à l'intérieur du macrocosme. Mais ce n'est là qu'un procédé parmi d'autres du portrait cosmique interactif.

\section{Figurer pour mieux activer}

En quoi le portrait bouscule-t-il ou, au contraire, renforce-t-il le dispositif de la consultation astrologique ordinaire? L'horoscope reste le cadre où, médiatisée par les astres, s'instaure la communication avec le client, ce dernier prenant conscience des courants qui le traversent et sur lesquels il peut agir par le moyen d'actes appropriés: rituels, port de pierres ou d'amulettes, etc. Autant de remèdes qui ne diffèrent pas de ceux que l'on peut rencontrer ailleurs en astrologie indienne ${ }^{9}$. Comme dans toute consultation astrologique, le consultant se retrouve entouré de tout un tas de présences astrales dotées de capacités d'influence que le pandit se charge de révéler. L'astrologue peuple l'environnement du consultant de petites forces agissantes derrière lui, au-dessus et à ses côtés. Mais l'acte de figuration ajoute autre chose à ce dispositif ordinaire.

"Pour comprendre ce qui se passe dans la tête d'un peintre, il suffit de suivre sa main", disait le cinéaste Henri-Georges Clouzot au début de son film, Le Mystère Picasso. Il est vrai que lorsqu'on observe un peintre au travail, on peut avoir l'impression que le cerveau œuvre à ciel ouvert, que les pensées intimes trouvent une manifestation visible ou une traduction concrète et immédiate sous la forme du tracé, bien davantage que dans toute autre activité. Suivre la main de l'astrologue en acte aide à lever ici une partie du mystère "astromorphologique ${ }^{\mathbf{1 0}}$ ». Prenons la technique de figuration qu'emploie le pandit pour tracer le portrait d'un mort ou d'une personne disparue, à la demande d'un commanditaire. Avant de mettre l'accent sur les caractères saillants de la personne, il propose au consultant assis en face de lui un portrait abstrait fait de points et de lignes. II aboutit à une sorte de «miroir fantomatique» qui est ensuite précisé peu à peu. Dans ce procédé que l'on peut appeler la technique de l'apparition progressive, le pandit a besoin de l'horoscope, mais aussi d'autres éléments et informations qui lui sont fournis par le consultant tout au long de la séance. Ceux-ci portent sur le caractère de la personne ou les événements qu'elle a traversés. Si l'horoscope ne peut être tenu pour le déterminant unique d'un visage, il permet de dessiner un certain nombre de lignes faibles (grand front, front plat, front concave) et de points qui constituent des nœuds d'accroche, rattachant le dessin à un cadre astrologique. Les traits fins, d'ordre anatomique, sont affinés au fur et à mesure des informations livrées par le consultant. L'astrologue arrive ainsi à dresser un portrait fidèle à l'image qu'en a le consultant, en "subsumant» les traits astraux aux étapes antérieures du dessin avant d'arriver progressivement au maximum de singularité. La main de l'astrologue active ou "éveille" certains points par le trait, ceux-là mêmes qui sont dérivés de l'horoscope. Lorsqu'un effet de ressemblance se produit, le client le voit et le dit. Kulkarni passe alors à une autre aire du visage, cherchant à aboutir à la même "étincelle de reconnaissance».

On se tromperait sur la nature du processus en pensant qu'un portrait singulier peut être entièrement composé à partir de l'horoscope. L'astrologue déplace son crayon d'une aire à l'autre du visage, créant des effets de ressemblance partiels (dont seul le consultant mesure l'efficacité) pour 

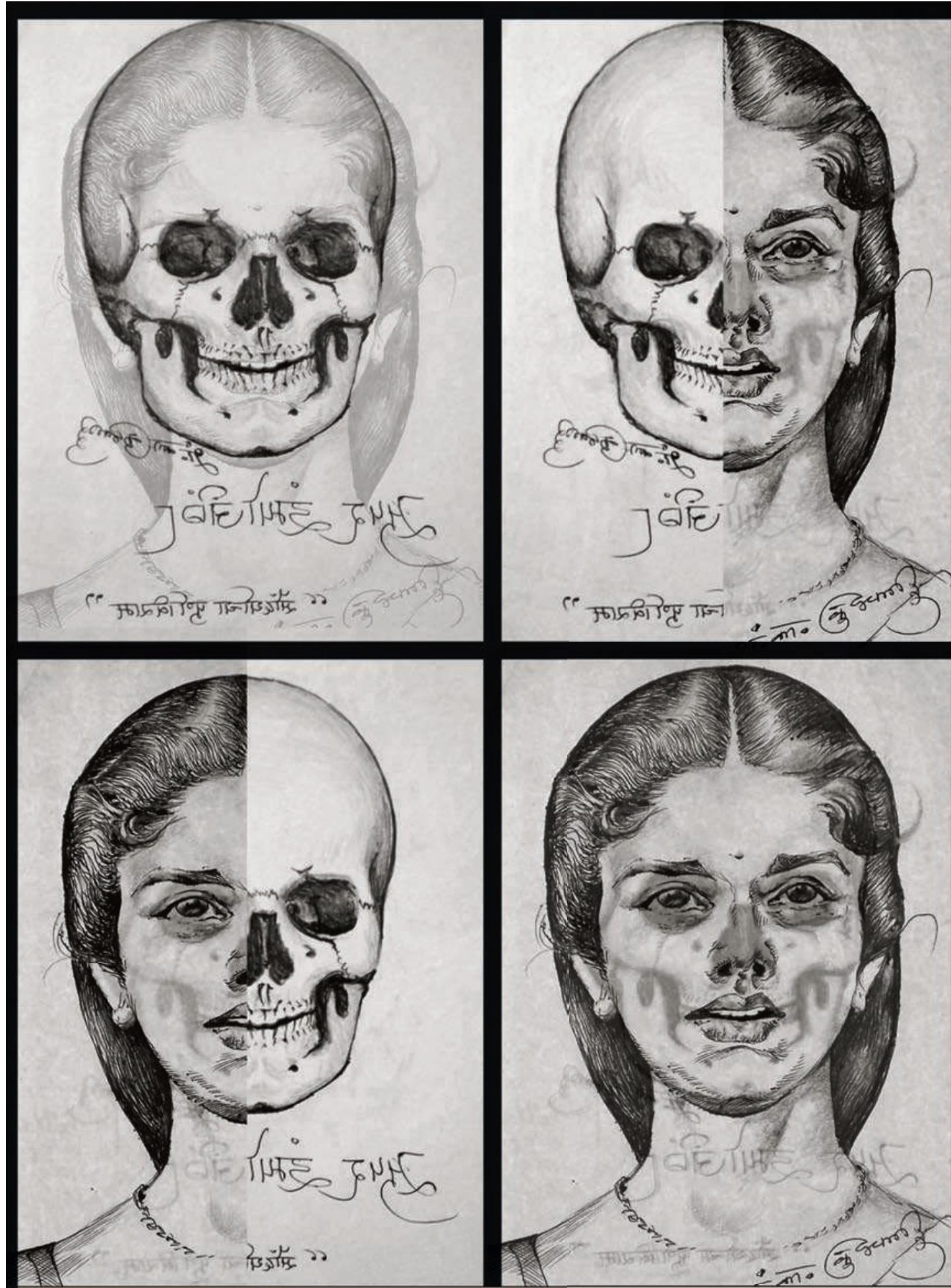

Elle a obtenu un diplôme de licence (BA). Elle est très belle, sensuelle et bien proportionnée. Nakshatra: Dhanishtha 3 Charan. Superposition. Le point final de la beauté. 
arriver peu à peu à une impression de ressemblance globale. Sans l'interaction avec le consultant, cette ressemblance ne peut donc pas advenir. Et sans point de départ horoscopique, il n'y aurait pas d'effet astral. Ce type de portrait n'a donc rien à voir avec un processus «magique» de dessin à l'aveugle. En revanche, il implique une grande finesse interactive, d'abord dans la manière dont il enrôle les astres dans la composition et ensuite dans la façon dont il fait participer le consultant à l'avènement du portrait. C'est en retournant aux points et lignes déjà effectués, par couches successives, en les infléchissant ou en les appuyant dans certaines directions à partir de ce que lui dit son client, éveillant ici et là des effets de ressemblance, que l'astrologue procède. En proposant des portraits génériques imprécis, il amène le consultant à fournir plus de précisions; sans qu'il ait l'impression d'en dire beaucoup, ce dernier apporte des informations déterminantes dans le processus d'achèvement. De ce point de vue, on peut parler ici d'un «paradoxe du trait révélateur » de la même façon qu'Arnaud Esquerre parle de «paradoxe de l'information révélatrice » à propos des consultations astrologiques dont il rend compte (Esquerre 2013). La main de Kulkarni semble avoir toujours un temps d'avance, être en «flux tendu» d'un point de vue temporel. Mais elle peut «rattraper le coup» et revenir en arrière en fonction des nouvelles informations fournies par le client dont toute l'attention est portée vers la montée en précision du portrait. Cette technique de "subsomption astrale " mériterait d'être comparée à d'autres techniques du portrait-robot qui posent des problèmes similaires dans la manière d'articuler "traits génériques» et recherche de la singularité, ressemblance et effet de reconnaissance. Dans tous les cas et même quand elles n'impliquent pas les astres, ces techniques tirent profit du fait que la ressemblance est un effet de perception (qui suppose quelqu'un pour la percevoir) et que la reconnaissance vient mettre un point d'arrêt à une recherche de singularité. La composition finit par se stabiliser dans un compromis entre le générique et le singulier. Cependant, il m'est arrivé très souvent de voir des clients se contenter d'une vague ressemblance. En effet, Kulkarni, comme tout artiste, a largement droit à l'erreur ou à l'imprécision, ce qui amène à penser que l'important dans ce genre de dessin est peut-être moins la précision finale que les étapes précédentes, lorsque le dessinateur subsume les traits astraux dans son dessin qui acquiert ainsi sa qualité de «portrait astral». Le consultant reconnaît toujours la difficulté technique que le peintre-astrologue doit surmonter et le fait d'avoir un portrait même un peu ressemblant est déjà en soi une prouesse. Ajoutons à cela que l'autorité de l'astrologue est telle qu'on ne va jamais remettre en cause son pouvoir de vision et que l'écart par rapport à la ressemblance est même souvent interprété comme étant le «style du pandit», la «touche de l'artiste».

\section{Puissance de l'astromorphisme}

On a dit à quel point le «portrait-robot» auquel aboutit l'astrologue constitue bien plus qu'un simple reflet rappelant au consultant les forces et les fardeaux dont son visage garde les traces. II a la potentialité d'infléchir (comme tout portrait artistique) l'image que cette personne a d'elle-même en en valorisant le mystère et en dramatisant les conflits, les paradoxes et les tensions qui l'animent. II nous reste à examiner comment s'opère cette astromorphose en intégrant les mécanismes de perception du patient. Certes, le pandit joue de son statut (de brahmane), de la proximité qu'il reven- 
dique avec les astres, de sa maîtrise du diagnostic et des attentes de son patient, mais ni plus ni moins que ne le ferait un médecin ou un autre spécialiste. Notons que, dans ses visualisations, il tombe souvent juste, mais même lorsque ce n'est pas le cas, l'échange ne tourne jamais à l'épreuve de vérité dans la mesure où la personne finit toujours par accepter une image, même non ressemblante, comme étant son portrait astral. Kulkarni est non seulement confiant mais autoritaire, il parle d'un ton assuré et rassurant, et grâce à sa personnalité il peut faire accepter n'importe quelle incongruité ou approximation comme un «effet de style».

Un exemple permettra d'illustrer ce point. Le pandit peut se servir du dessin pour représenter l'emprise d'une planète à un moment donné, la figeant dans une «expression révélatrice». Un jour, une femme et sa fille viennent le consulter pour se plaindre du comportement du père, un être violent, sans travail et ayant, entre-temps, abandonné le domicile familial. Le pandit demande à la femme s'ils se battent souvent. Elle répond par la négative. L'homme est parti parce qu'il a perdu son emploi. Le pandit trace le portrait de celui-ci à partir de son horoscope et des informations fournies par les deux consultantes. II en fait un portrait presque caricatural à mon goût, mais l'expression du visage tombe juste du point de vue des consultantes. L'une d'elles dit: “C'est souvent en effet ainsi qu'il me regarde. C'est bien lui ! ” Dans le cabinet du pandit, la figuration d'un état émotionnel ou d'une attitude prototypique l'emporte souvent sur celle des détails du visage. Kulkarni est très doué pour incarner dans un visage des émotions et des états fort subtils, demandant alors au client de juger l'«expression », le «style». L'intérêt de telles séances, par rapport à d'autres consultations d'astrologues qui ne recourent pas au dessin, est que la personne absente faisant l'objet d'un diagnostic ou d'une prédiction devient présente de manière bien plus incarnée que lorsqu'on se contente de la convoquer par le langage. Dans l'exemple qui nous occupe, ce que les consultantes veulent surtout savoir est si le père rentrera chez lui et quand. Le pandit leur répond qu'il ne reviendra plus, que son usine souffrira de pertes énormes et qu'il a perdu la tête. «Voyez comme il est sous l'emprise de Saturne!» dit Kulkarni en brandissant le portrait. Et l'homme n'a effectivement jamais réapparu bien que la police ait lancé plusieurs avis de recherche. Un an après, quand je suis revenu voir le pandit, I'homme était toujours porté disparu. Le pandit l'avait prévu: “Cet homme doit aller traiter sa folie quelque part. C'est écrit dans son horoscope!» Ainsi le pandit, comme tout astrologue indien, sert-il de confident privilégié dans la gestion des conflits et des drames familiaux. L'astrologue réalise la conversion des situations terriennes en drames cosmiques avec une assurance troublante et avec la double complicité des astres et du dessin.

\section{Conclusion}

C'est avec le souci du chercheur que Kulkarni a accumulé toute sa vie des données sur les visages les plus divers. Son astromorphologie part du principe que ces corps influencés et influençables sont un terrain de jeu favori pour les planètes, qui agissent ici par poussées et pressions à des fréquences inaccessibles à la perception ordinaire. Ces emprises posant un problème de représentation ou de visibilité, on mesure ici tout l'intérêt de l'expérimentation picturale. Soulignons qu'à aucun moment l'astrologue ne semble se mettre en position transcendante. II s'inclut toujours dans la chaîne psycho- 


\section{एव्चीच}

प्रेम कुमार शर्मा

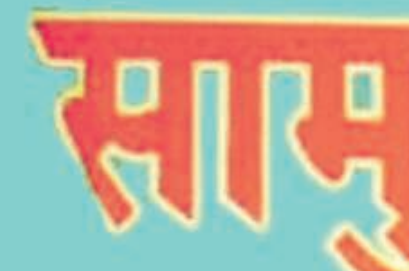

$\infty$

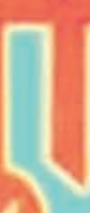

$\left.\lim _{10} \sqrt{6}\right)$
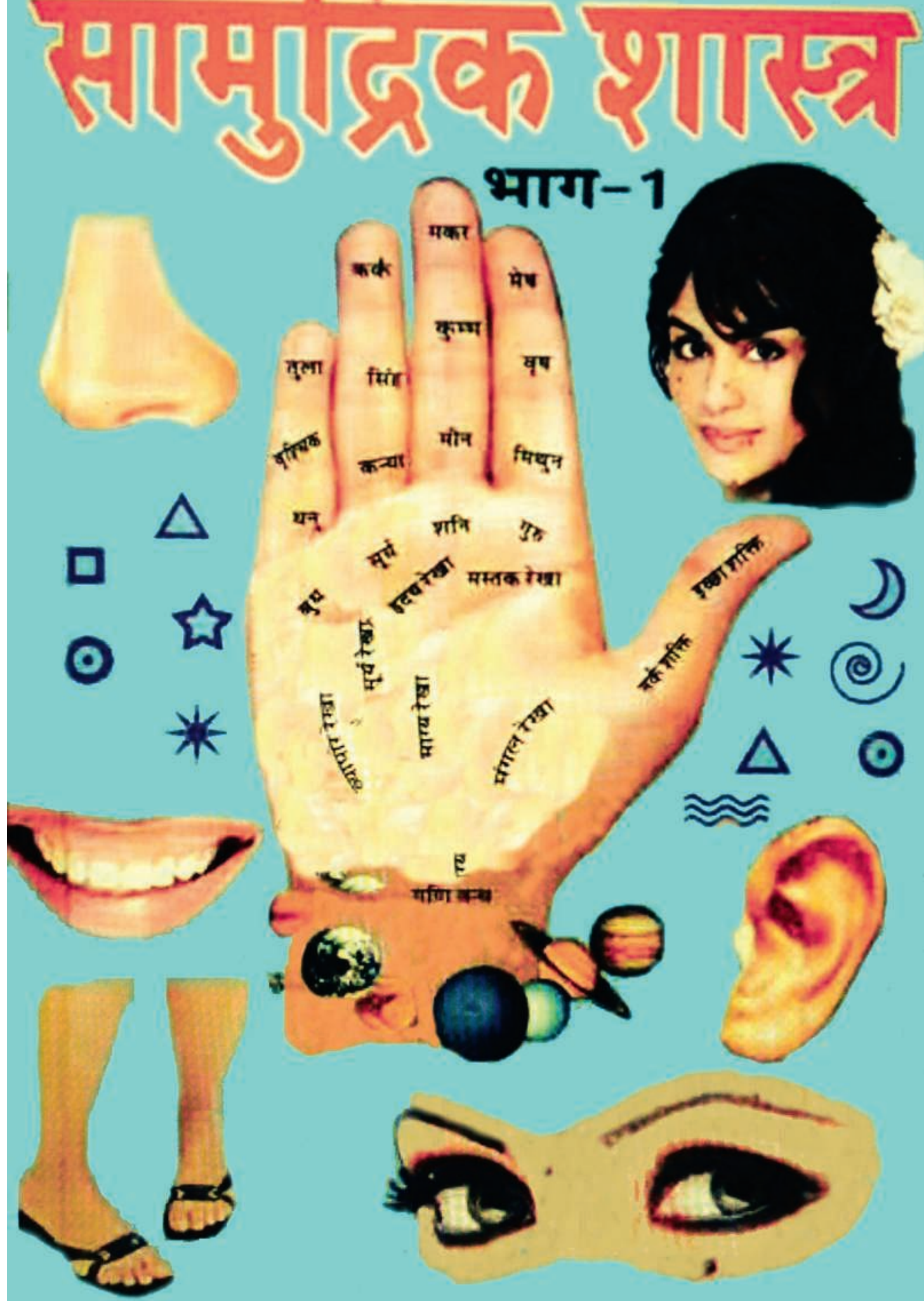

थन् शनि उ० बै के मसक रेखा

-1

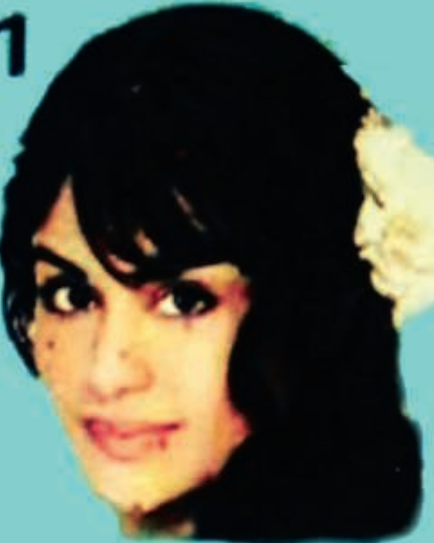

口 4 $\mathrm{c}^{\mathrm{x}^{2}}$ मस्तकरेखा

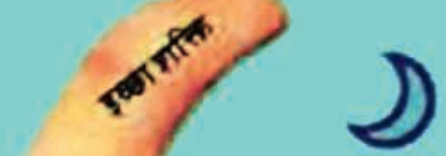
ह $x^{*} \frac{1}{4}$

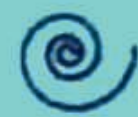<smiles>C=CC(=C)C=C</smiles>

bist

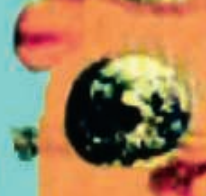

$\Delta$

(a)

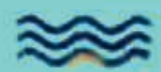


physique de transmission des emprises. II faut insister sur ce point: penser suffit parfois à être influencé. Dans l'exemple précédent, l'expression de folie représentée dans le portrait et identifiée par les consultantes ne pouvait qu'être expliquée par l'influence de Saturne dans la mesure où, durant l'acte même de figuration, l'astrologue traçait l'esquisse sous l'emprise de cette planète. Si la matrice astrale habitait donc ce visage, comme tous les autres, le fait que l'astrologue puisse se faire ici la main de Saturne (comme il peut se faire la main d'autres planètes, selon la situation du consultant) montre qu'il n'y a aucune contradiction à penser son dessin comme étant à la fois un processus d'activation/d'éveil de liaisons astrales et un acte de représentation, à partir du moment où les «effets de style" sont perçus comme des connexions en acte dans l'interaction avec le consultant.

Faisant valoir que les étoiles sont beaucoup trop éloignées de la planète Terre pour exercer sur ses habitants la moindre pression et dénonçant, de ce fait, le caractère non scientifique de l'astrologie, Prakash Deshpande, physicien indien, fait la remarque suivante: "Sous l'étiquette d'astrologie, les astrologues pratiquent une sorte de traitement psychiatrique sur des bases astrologiques. C'est un genre de consultation qui aide à agir. Les astrologues sont les hommes les plus influents de ce pays, même si leur savoir n'a jamais été statistiquement prouvé ${ }^{11}$. " Deshpande ne fait en réalité que reproduire la critique adressée depuis longtemps à l'astrologie en Occident. Il est évident en effet que faire de celle-ci une branche des savoirs «psychiques» plutôt qu'une branche des «sciences naturelles» la rend plus acceptable aux yeux des sceptiques. Ce débat continue malheureusement à miner toute discussion portant sur l'astrologie alors que son originalité est ailleurs: dans les enchaînements psychophysiques qu'elle propose où tout corps peut devenir vecteur de transmission d'une emprise, qu'il s'agisse d'objets matériels, d'images, d'actes ou de pensées.

Dans ce contexte, on ne peut se contenter de dire que l'astrologie, en faisant parler les astres, ne fait que jouer sur des ressorts psychologiques. Les astrologues doivent par ailleurs rendre des comptes à ceux qui les consultent. Les expériences de quantification des présences astrales auxquelles ils s'adonnent ne sont pas seulement les leurs. Sans atteindre à un minimum de résonance dans la vie même de leur client, leur diagnostic perd toute crédibilité. À ce titre, le portrait propose une nouvelle expérience de diffraction et offre une autre chance d'éprouver les résonances. C'est que le cosmos des astrologues n'est pas ce ciel qui trône au-dessus de nos têtes, destiné à être contemplé de loin tel un paysage. La chorégraphie capricieuse des astres est faite pour être vécue et la matrice astrale, cet univers dans lequel le Terrien navigue et qui n'est rien d'autre que son monde, ressentie dans sa propre chair. Dans l'histoire des techniques, les dispositifs permettant de faire de telles expériences de connexion entre d'un côté les humains et de l'autre les entités les plus éloignées qui puissent exister ne sont pas si nombreux. II faut bien reconnaître à l'astrologie cette vertu: elle n'a jamais cessé de chercher, avec plus ou moins d'inventivité et de force de conviction, à combler ce grand écart.

CNRS-Laboratoire d'ethnologie et de sociologie comparative emmanuel.grimaud@gmail.com
11. Extrait d'une interview de Prakash Deshpande réalisée par l'auteur en 2003 à Poona.

\footnotetext{
ci-contre

fig. 8

Traité de chiromancie populaire (samudrika shastra), par Prem Kumar Sharma, Delhi, DPB Publishing, 2009.
} 


\section{Avdeeff, Alexis}

2014 Les Feuilles de palme et le stylet. L'art de la prédiction astrologique chez les Valluvar du pays tamoul (Inde du Sud). Université de Toulouse-Le Mirail, thèse de doctorat.

\section{Binet, Alfred}

1887 «L'intensité des images mentales", Revue philosophique de la France et de l'étranger: 473-497.

\section{Bruno, Giordano \\ 2001 [1591] Des liens. \\ Paris, Allia.}

\section{Cardan, Jérôme}

2010 [1558] La Métoposcopie. Des observations des lignes du front des femmes. Des seings, ou marques naturelles du visage, et des lignes qui les accompagnent. Paris, Alain Baudry.

\section{Curry, Patrick (dir.)}

1987 Astrology, Science and Society: Historical Essays. Woodbridge, Boydell and Brewer.

\section{Das, Goravani}

1997 "Choice of authority in vedic astrological software ", in Richard Houck (éd.), Hindu Astrology Lessons. Gaithersburg, Groundswell Press.

\section{DeFouw, Hart}

\section{et Svoboda, Robert}

1996 Light on Life. An Introduction to the Astrology of India. New Delhi, Penguin Books.

\section{Drévillon, Hervé}

1996 Lire et écrire l'avenir: l'astrologie dans la France du Grand Siècle, 1610-1715.

Seyssel, Champ Vallon.

\section{Dutta, Prajit K.}

1999 Strategies and Games: Theory and Practice. Cambridge, The MIT Press.

\section{Esquerre, Arnaud}

2013 Prédire. L'astrologie en France au $x x 1^{\ominus}$ siècle. Paris, Fayard.

\section{Fechner, Gustav}

1997 [1825] Anatomie comparée des anges. Paris, Éditions de l'Éclat.

\section{Gall, Franz Joseph}

1810 Anatomie et Physiologie du système nerveux en général et du cerveau en particulier avec des observations sur la possibilité de reconnaitre plusieurs dispositions intellectuelles et morales de l'homme et des animaux par la configuration de leurs têtes. Paris, Schoell.

\section{Grafton, Anthony}

1994 Cardano's Cosmos. Harvard, Harvard University Press.

\section{Grimaud, Emmanuel}

2014 L'Étrange Encyclopédie du docteur K. Portraits et horoscopes d'un astrologue indien. Paris, Société d'ethnologie.

\section{Guenzi, Caterina}

2013 Le Discours du destin. La pratique de l'astrologie à Bénarès. Paris, CNRS Éditions.

\section{Liberski-Bagnoud, Danouta}

2012 "La chorégraphie du bâton divinatoire comme écriture sonore au Burkina Faso ", Gradhiva (n.s.) 15: 182-201.

\section{Mayer, Andreas}

2013 Sites of the Unconscious: Hypnosis and the Emergence of the Psychoanalytic Setting. Chicago, University of Chicago Press.

\section{Minkowski, Christopher}

2002 «Astronomers and their reasons: Working paper on Jyotihśāstra ", Journal of Indian Philosophy 30(5) : 495-514.

\section{Peirce, Charles Sanders}

1878 «Comment se fixe la croyance", Revue philosophique de la France et de l'étranger VI.

\section{Pingree, David}

1965 «Representations of the planets in Indian astrology ", Indo-Iranian Journal 8: 249-267.

\section{Pugh, Judith}

1980 «Person and experience: The astrological system of North India». Université de Chicago, thèse de doctorat.

1983a "Astrological counseling in contemporary India ", Culture, Medicine and Psychiatry 7(3): 279-299.

1983b "Astrology and fate ", in Charles F. Keyes et E. Valentine Daniel (éd.), Karma:

An Anthropological Inquiry.

Berkeley, University of California Press: 131-146.

1984 "Concepts of person and situation in North Indian counseling: The case of astrology ", Contributions to Asian Studies 18: 85-105.

1988 «Divination and ideology in the Banaras muslim community ", in Katherine P. Ewing (dir.), Shariat and ambiguity in South Asian Islam. Berkeley, University of California Press: 288-306.

\section{Raymond, Joad (dir.)}

2011 Conversations with Angels: Essays towards a History of Spiritual Communication, 1100-1700. Basingstoke, Palgrave Macmillan.

\section{Renneville, Marc}

2000 Le Langage des crânes. Une histoire de la phrénologie. Paris, Synthélabo.

\section{Renou, Louis}

1925 «Connexion en védique, cause en bouddhique ", in L'Inde fondamentale. Paris, Hermann: 149-153.

\section{Schaffer, Simon}

2011 "Newtonian angels ", in Raymond (dir.) 2011.

\section{Simon, Gérard}

1992 Kepler, astronome, astrologue. Paris, Gallimard.

\section{Tarabout, Gilles}

2002 "Les corps et les choses. Résonances et métaphores corporelles dans l'astrologie appliquée aux temples (Kérala) », in Véronique Bouillier et Gilles Tarabout (éd.), Images du corps dans le monde hindou. Paris, CNRS Éditions ("Monde indien»): 135-159.

2006 "La réparation des fautes. Le contrôle astrologique de la transformation des rites et des temples au Kérala ", in Gérard Colas et Gilles Tarabout (éd.), Rites hindous: transferts et transformations. Paris, EHESS («Purusartha» 25) : 463-493.

\section{Vaught, Louis Allen}

1908 [1902] Lecture pratique du caractère. Bruxelles, Institut de culture humaine (édition originale anglaise Vaught's practical character reader).

\section{Von Neumann, John} et Morgenstern, Oskar

1944 Theory of Games and Economic Behaviours. Princeton, Princeton University Press (Théorie des jeux et comportements économiques, université des sciences sociales de Toulouse, 1977, pour la traduction française)

\section{Zeytlin, David}

1990 «Professor Garfinkel visits the soothsayers. Ethnomethodology and Mambila divination ", Man 25(4): 654-666.

1993 « Spiders in and out of court or "the long legs of the law": Styles of spider divination in their sociological contexts ", Africa 63(2): 219-240.

2012 " Divinatory logics: Diagnoses and predictions mediating outcomes", Current Anthropology 53(5) : 525-546. 
